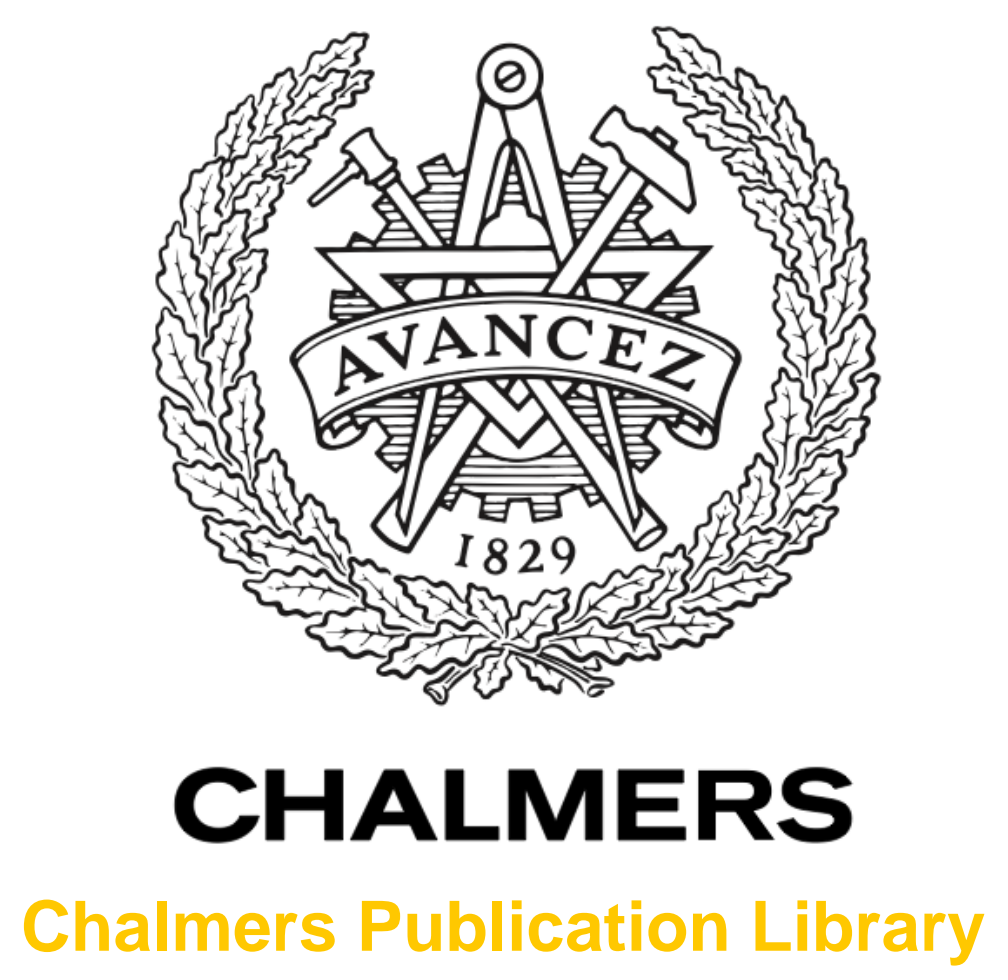

\title{
On the Trade-Off Between Accuracy and Delay in Cooperative UWB Localization: Performance Bounds and Scaling Laws
}

This document has been downloaded from Chalmers Publication Library (CPL). It is the author's version of a work that was accepted for publication in:

IEEE Transactions on Wireless Communications (ISSN: 1536-1276)

Citation for the published paper:

Garcia, G. ; Muppirisetty, L. ; Schiller, E. et al. (2014) "On the Trade-Off Between Accuracy and Delay in Cooperative UWB Localization: Performance Bounds and Scaling Laws". IEEE Transactions on Wireless Communications, vol. 13(8), pp. 4574-4585.

http://dx.doi.org/10.1109/TWC.2014.2314662

Downloaded from: http://publications.lib.chalmers.se/publication/205773

Notice: Changes introduced as a result of publishing processes such as copy-editing and formatting may not be reflected in this document. For a definitive version of this work, please refer to the published source. Please note that access to the published version might require a subscription. 


\title{
On the Trade-off Between Accuracy and Delay in Cooperative UWB Localization: Performance Bounds and Scaling Laws
}

\author{
Gabriel E. Garcia, Student Member, IEEE, L. Srikar Muppirisetty, Elad M. Schiller, Member, IEEE \\ and Henk Wymeersch, Member, IEEE
}

\begin{abstract}
Ultra-wide bandwidth (UWB) systems allow for accurate positioning in environments where global navigation satellite systems may fail, especially when complemented with cooperative processing. While cooperative UWB has led to centimeter-level accuracies, the communication overhead is often neglected. We quantify how accuracy and delay trade off in a wide variety of operation conditions. We also derive the asymptotic scaling of accuracy and delay, indicating that in some conditions, standard cooperation offers the worst possible tradeoff. Both avenues lead to the same conclusion: indiscriminately targeting increased accuracy incurs a significant delay penalty. Simple countermeasures can be taken to reduce this penalty and obtain a meaningful accuracy/delay trade-off.
\end{abstract}

Index Terms-Ultra-wideband positioning, S-TDMA, MAC delay, navigation, positioning

\section{INTRODUCTION}

$\mathbf{P}$ OSITION information is a necessary part of today's location-aware applications [3], including inventory tracking in warehouses [4], habitat [5] and health monitoring [6]. Even though Global Navigation Satellite Systems (GNSSs) can help to provide position information in many situations, they may not be viable in weak signal environments such as urban canyons, or indoors [7]. In consequence, there exists an ongoing need for accurate positioning in scenarios where GNSS-only implementations are not feasible.

Ultra-wide bandwidth (UWB) ranging and communication has been shown to be a promising technology to tackle the positioning problem in GPS-challenged scenarios. This pulsebased technology [8] offers a range of characteristics to avail of, both in terms of communication and localization. UWB communication advantages include robustness against interference and mitigation of small-scale fading [9]. Moreover, considering a two-way time of-arrival (TW-TOA) ranging procedure, UWB enables accurate and reliable ranging, making it convenient for localization and navigation purposes [10],

G. Garcia, L.S. Muppirisetty and H. Wymeersch are with the Department of Signals and Systems, Chalmers University of Technology, Gothenburg, Sweden, e-mails: \{ggarcia,srikar.muppirisetty,henkw\} @ chalmers.se. E.M. Schiller is with the Department of Computer Science and Engineering, Chalmers University of Technology, Gothenburg, Sweden, e-mail: elad.schiller@chalmers.se. This research was supported, in part, by the European Research Council, under Grant No. 258418 (COOPNET), the Swedish Research Council, under Grant No. 2010-5889, and by the EC, through project FP7-STREP-288195, KARYON (Kernel-based ARchitecture for safetY-critical cONtrol). Part of this work has been presented at IEEE Wireless Communications and Networking Conference 2013 [1], and published in IEEE Communication Letters [2].
[11]. Improving positioning accuracy has gathered a significant amount of attention in the research community. The general conclusion is that traditional methods, such as enhanced ranging [7], the use of more anchors, higher transmission powers [12], and cooperation among nodes [13]-[17], all improve the positioning accuracy.

In practice, performance gains in terms of accuracy come at a cost in delay, due to the channel access required in the medium access control (MAC) layer. This cost, which was neglected in [7], [12]-[17], has been analyzed in [18], [19], for cooperative positioning and target tracking, respectively, though based solely on computer simulations. Moreover, the IEEE 802.11.b MAC considered in [18] leads to excessively pessimistic delays. Dedicated MAC protocols were discussed in [20]-[24]: a decentralized self-stabilizing MAC protocol suitable for cooperative UWB navigation in multi-hop networks was proposed in [20], while in [21] and [22], a distributed and decentralized scheduling for cooperative localization was explored. A MAC design for cooperative localization networks was investigated in [23], focusing exclusively on the analysis and design of the MAC protocol. Finally, [24] proposed enhancements to the IEEE 802.15.4a standard using a time division multiple access (TDMA) scheme for networks with complete communication graphs.

In this paper, we tackle the trade-off between accuracy and delay through performance bounds. First, we derive lower bounds on UWB positioning accuracy in terms of the position error bound (PEB) [12] and on the required MAC delay. We also evaluate two methods to reduce the delay: selective ranging [25] and eavesdropping [15]. Finally, we derive scaling laws for the PEB and MAC delay for several scenarios. Our specific contributions are as follows:

- A derivation of the PEB and minimum MAC delay for noncooperative and cooperative networks with selective ranging and eavesdropping, assuming spatial reuse TDMA (STDMA) and two-way ranging as an extension to [1], [2], where eavesdropping was only considered for noncooperative networks;

- Scaling laws on the positioning accuracy and minimum MAC delay for dense networks, for several distinct operating conditions; and

- The introduction of a delay/accuracy trade-off parameter, which can uniquely quantify the trade-off between PEB and MAC delay as a function of the agent and anchor density. 


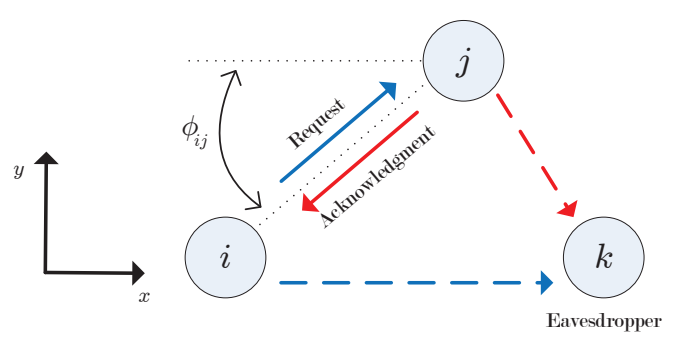

Figure 1. Two-dimensional graphic representation of the TW-TOA ranging transaction between nodes $i$ and $j$, while node $k$ performs an eavesdropping measurement.

The remainder of the paper is structured as follows. Section II presents the measurement and network models for UWB positioning. In Section III, we review the derivation of the lower bound on positioning accuracy and minimum MAC delay. Then, in Section IV we derive the scaling laws for the positioning accuracy and the MAC delay. Finally, numerical results are given in Section $\mathrm{V}$, followed by the conclusions in Section VI.

\section{SySTEM MODEL}

\section{A. UWB Positioning}

We consider a wireless network consisting of $M$ anchor nodes (collected in the set $\mathcal{S}_{\text {anchors }}$ ) with known positions and $N$ agent nodes (collected in the set $\mathcal{S}_{\text {agents }}$ ) with unknown time-varying positions. The agents move in discrete time slots of duration $T$, and we focus on a specific time slot, at which the position of node $i$ is denoted by $\mathbf{x}_{i}=\left[x_{i} y_{i}\right]^{\mathrm{T}}$, with an a priori distribution $p\left(\mathbf{x}_{i}\right)$. Before moving on to the next time slot, the agent corrects its distribution to an a posteriori distribution $p\left(\mathbf{x}_{i} \mid \mathbf{z}_{i}\right)$, where $\mathbf{z}_{i}$ represents the available measurements. The time slot duration $T$ is lower bounded by the measurement time $T_{\text {meas }}$, required by the agents to gather all the UWB measurements. We aim to quantify how traditional methods to improve accuracy, such as adding more anchors or agents, increasing the transmission power by means of the communication range, and the employment of cooperation among the agents affect the position accuracy (see Section III) and $T_{\text {meas }}$ (see Section III.C). Note that $T$ also comprises other components, such as a computation time and possibly a data exchange time. Since these operations may be performed with an alternative radio technology, these times are not strictly UWB-related, and are thus not included in our study. Moreover, dedicated methods to reduce the delay can also be derived for those operations [26], [27].

\section{B. Measurement Models}

We consider two types of UWB measurements: twoway time-of-arrival (TW-TOA) and eavesdropping, shown in Fig. 1. In a TW-TOA transaction, agent $i$ sends a request to node $j$, which responds back with an acknowledgment. The set of neighbors of node $i$ is denoted by $\mathcal{N}_{i}=\{j \neq i: i$ and $j$ can communicate $\}$. When $j \in \mathcal{S}_{\text {agents }} \cup \mathcal{S}_{\text {anchors }}$, we say that the network is cooperative, while when $j$ is constrained to belong to $\mathcal{S}_{\text {anchors, }}$, we say that the network is noncooperative. In either case, both nodes $i$ and $j$ estimate the TOA for the request and acknowledgment, respectively. Agent $i$ employs the round trip delay between itself and node $j$ to estimate their distance. The TW-TOA measurement between agent $i$ and node $j$ is given by [15]:

$$
z_{i j}=\underbrace{d_{i j}+\frac{c T_{\mathrm{proc}}}{2}}_{=\mu_{i j}}+\frac{n_{i j}}{2}+\frac{n_{j i}}{2}
$$

where $d_{i j}=\left\|\mathbf{x}_{i}-\mathbf{x}_{j}\right\|, n_{i j}$ is the TOA error of the request from node $i$ to node $j$ and $n_{j i}$ is the TOA error from the acknowledgment from node $j$ to node $i, c$ is the speed of light, and $T_{\text {proc }}$ is a known processing time. The TOA errors are modeled as independent zero-mean Gaussian random variables: $n_{i j} \sim \mathcal{N}\left(0, \sigma_{i j}^{2}\right)$ and $n_{j i} \sim \mathcal{N}\left(0, \sigma_{j i}^{2}\right)$. For the eavesdropping measurements, any node $k \in \mathcal{N}_{i} \cap \mathcal{N}_{j}$ is able to measure the TOA of the signals exchanged between nodes $i$ and $j$. We obtain the eavesdropping measurement by subtracting those two TW-TOA measurements [15]:

$$
z_{i j}^{k}=\underbrace{d_{i j}+d_{j k}-d_{i k}+c T_{\text {proc }}}_{=\mu_{i j}^{k}}+n_{i j}+n_{j k}-n_{i k} .
$$

It is important to note that there exists a common noise term between (1) and (2), since one of the TOA measurements collected by node $k$ depends on the TOA measurement of node $j$.

Under line-of-sight conditions, the ranging error variance between two nodes at a distance $d$ apart can be modeled as in [13]:

$$
\sigma^{2}(d)= \begin{cases}\sigma^{2} & d \leq R_{\mathrm{hw}} \\ \sigma^{2} f(d) & R_{\mathrm{hw}}<d \leq R_{\max } \\ +\infty & d>R_{\max },\end{cases}
$$

where $R_{\mathrm{hw}}$ is the range for which the variance is dominated by the hardware (e.g., ADC, filters), $R_{\max }$ is the maximum communication range, and $f(d)$ is a non-decreasing function with $f\left(R_{\mathrm{hw}}\right)=1$, capturing the degradation of the signal-to-noise ratio or the ranging information intensity [12]. Based on our off-the-shelf UWB hardware [28] and previous experiments [13], $R_{\mathrm{hw}}$ can be around 30 meters, which is sufficient for many indoor environments. In this paper, we limit ourselves to this range, i.e., $d \in\left(0, R_{\mathrm{hw}}\right]$.

\section{Network Model}

We assume that nodes $i$ and $j$ can communicate with probability $P_{i j}=\exp \left(-\left\|\mathbf{x}_{i}-\mathbf{x}_{j}\right\|^{2} /\left(2 R^{2}\right)\right)$, where $R$ is the nominal communication range in meters, as in [29]. Node $i$ can perform UWB measurements with any node in $\mathcal{N}_{i}$. For increased flexibility, we introduce the set $\mathcal{S}_{i} \subseteq \mathcal{N}_{i}$, which consists of selected neighbors with which node $i$ performs TW-TOA ranging.

When two TW-TOA transactions are performed simultaneously, they can interfere if a node in one transaction can receive a packet from a node in the other transaction. As 802.15.4a radios use a common preamble, similar to our offthe-shelf radios, we do not rely on time hopping to deal 
with interference. Traditional protocols such as ALOHA or slotted ALOHA [30] have poor efficiency in terms of the successful number of transactions. Hence, similar to [24], [31], we consider an STDMA approach in which one TDMA slot is needed for a TW-TOA transaction, but two transactions can occur simultaneously if they do not interfere. The total STDMA delay within one time slot ${ }^{1}$ depends only on the network topology in the current time slot. In contrast to [24] and [31], we do not consider ranging packet aggregation or other enhancements, as they are hard to justify with real hardware due to the tight synchronization constraints.

\section{LOWER BOUND ON POSITIONING ACCURACY AND MAC DELAY}

In this section, we will derive a generic expression for the PEB for both TW-TOA and eavesdropping measurements, for both cooperative and noncooperative networks. We first review the concepts of Fisher information matrix (FIM), equivalent Fisher information matrix (EFIM), and PEB. We end with a short discussion on bounds on the minimum MAC delay.

\section{A. PEB: Basic Concepts}

Let $\mathbf{x}=\left[\mathbf{x}_{1}^{\mathrm{T}} \mathbf{x}_{2}^{\mathrm{T}} \cdots \mathbf{x}_{N}^{\mathrm{T}}\right]^{\mathrm{T}}$ be the vector containing the positions of all agents and $\hat{\mathbf{x}}$ its estimate, based on the observation $\mathbf{z}$. The FIM is defined as $\mathbf{J}=-\mathbb{E}_{\mathbf{x}, \mathbf{z}}\left\{\nabla_{\mathbf{x}} \nabla_{\mathbf{x}}^{\mathrm{T}} \log p(\mathbf{z}, \mathbf{x})\right\}$ for random $\mathbf{x}$, and as $\mathbf{J}(\mathbf{x})=-\mathbb{E}_{\mathbf{z}}\left\{\nabla_{\mathbf{x}} \nabla_{\mathbf{x}}^{\mathrm{T}} \log p(\mathbf{z} \mid \mathbf{x})\right\}$ for nonrandom $\mathbf{x}$ [32], where $\nabla_{\mathbf{x}}$ denotes the derivative with respect to $\mathbf{x}$. We will drop the argument $\mathbf{x}$ in the FIM as it will be clear from the context whether the variable is random or not. The EFIM of the first agent is defined as follows. Let

$$
\mathbf{J}=\left[\begin{array}{cc}
\mathbf{A} & \mathbf{B} \\
\mathbf{B}^{\mathrm{T}} & \mathbf{C}
\end{array}\right]
$$

where $\mathbf{A} \in \mathbb{R}^{2 \times 2}$, $\mathbf{B} \in \mathbb{R}^{2 \times 2(N-1)}$, and $\mathbf{C} \in$ $\mathbb{R}^{2(N-1) \times 2(N-1)}$, then the EFIM for agent 1 is given by $\mathbf{J}_{1}^{\mathrm{E}}=\mathbf{A}-\mathbf{B C}^{-1} \mathbf{B}^{\mathrm{T}}$ [12] . Using the Schur complement it is easy to verify that $\left[\mathbf{J}_{1}^{\mathrm{E}}\right]^{-1}$ is the top-left $2 \times 2$ block diagonal element of $\mathbf{J}^{-1}$. The EFIM for any agent $i$ can be computed through a reordering of the agents. A similar definition of the EFIM holds for random $x$. Finally, the PEB of the network is defined as $\mathcal{P}=\sqrt{\operatorname{tr}\left\{\mathbf{J}^{-1}\right\} / N}$, while the PEB of agent $i$ is defined as $\mathcal{P}_{i}=\sqrt{\operatorname{tr}\left\{\left[\mathbf{J}_{i}^{\mathrm{E}}\right]^{-1}\right\}}$. From the theory of the Crámer-Rao lower bound (CRLB) [32], it is well known that, under suitable technical conditions, $\mathcal{P}^{2} \leq \mathbb{E}\left\{\|\mathbf{x}-\hat{\mathbf{x}}\|^{2}\right\}$, and $\mathcal{P}_{i}^{2} \leq \mathbb{E}\left\{\left\|\mathbf{x}_{i}-\hat{\mathbf{x}}_{i}\right\|^{2}\right\}$, where the expectation should be taken of the relevant random variables.

Note that the PEB is expressed in meters and that $\mathcal{P}$ and $\mathcal{P}_{i}$ are related through $\mathcal{P}=\sqrt{\sum_{i} \mathcal{P}_{i}^{2} / N}$.

\section{B. PEB: Derivation}

We again collect the positions of all agents in a vector $\mathbf{x}$ and determine the FIM. For mathematical convenience

${ }^{1}$ Note that we make a distinction of time slots of duration $T$, which capture the slow time scale of mobility, and much shorter TDMA slots, in which multiple ranging transactions are scheduled. and since we are focusing on a single time slot, we assume that every agent has an a priori distribution $p\left(\mathbf{x}_{i}\right)$, modeled as a symmetric Gaussian distribution with mean $\mathbf{m}_{\text {prior }, i}$ and variance $\sigma_{\text {prior }, i}^{2}$ per dimension. Recalling the definition of the set $\mathcal{S}_{i} \subseteq \mathcal{N}_{i}$, we construct a measurement vector $\mathbf{z}$ for both the noncooperative and cooperative cases as $\mathbf{z}=\left[\mathbf{z}_{i j} \mid i \in \mathcal{S}_{\text {agents }}, j \in \mathcal{S}_{i}\right]$, where $\mathbf{z}_{i j}$ contains the TWTOA estimate between agent $i$ and node $j$, as well as all the corresponding eavesdropping measurements at node $k$, $\mathbf{z}_{i j}=\left[z_{i j},\left\{z_{i j}^{k} \mid k \in \mathcal{N}_{i} \cap \mathcal{N}_{j}\right\}\right]$.

Due to (1) and (2), $\mathbf{z}$ conditioned on $\mathbf{x}$ is a Gaussian random variable with mean $\mu$, constructed from (1) and (2) in the same way as $\mathbf{z}$, and covariance matrix $\boldsymbol{\Sigma}$. As a result of the independence of the TW-TOA measurements, the covariance matrix $\Sigma$ is a block diagonal matrix, with the block corresponding to $\mathbf{z}_{i j}$ given by

$$
\mathbf{C}_{i j}=\mathbb{E}\left\{\left(\mathbf{z}_{i j}-\boldsymbol{\mu}_{i j}\right)\left(\mathbf{z}_{i j}-\boldsymbol{\mu}_{i j}\right)^{\mathrm{T}}\right\},
$$

where the entries are given by $\mathbb{E}\left\{\left(z_{i j}-\mu_{i j}\right)^{2}\right\}=\left(\sigma_{i j}^{2}+\sigma_{j i}^{2}\right) / 4$, $\mathbb{E}\left\{\left(z_{i j}-\mu_{i j}\right)\left(z_{i j}^{k}-\mu_{i j}^{k}\right)\right\}=\sigma_{i j}^{2} / 2$, and

$$
\mathbb{E}\left\{\left(z_{i j}^{l}-\mu_{i j}^{l}\right)\left(z_{i j}^{k}-\mu_{i j}^{k}\right)\right\}= \begin{cases}\sigma_{i j}^{2}+\sigma_{j k}^{2}+\sigma_{i k}^{2} & k=l \\ \sigma_{i j}^{2} & k \neq l .\end{cases}
$$

Note that when there are only TW-TOA measurements, $\mathbf{C}_{i j}$ reverts to the scalar $\left(\sigma_{i j}^{2}+\sigma_{j i}^{2}\right) / 4$. The FIM is now given by [33]

$$
\mathbf{J}=-\mathbb{E}_{\mathbf{x}}\left\{\nabla_{\mathbf{x}} \boldsymbol{\mu}^{\mathrm{T}} \boldsymbol{\Sigma}^{-1} \nabla_{\mathbf{x}}^{\mathrm{T}} \boldsymbol{\mu}\right\}+\mathbf{J}_{\text {prior }}
$$

where $\mathbf{J}_{\text {prior }}=\operatorname{diag}\left[\sigma_{\text {prior }, 1}^{2}, \sigma_{\text {prior }, 1}^{2}, \ldots, \sigma_{\text {prior }, N}^{2}, \sigma_{\text {prior }, N}^{2}\right]^{-1}$. The entries in the matrix $\nabla_{\mathbf{x}} \boldsymbol{\mu}^{\mathrm{T}}$ can be easily computed since they are all zero, except for $\partial \mu_{i j} / \partial \mathbf{x}_{i}=\left[\cos \left(\phi_{i j}\right) \sin \left(\phi_{i j}\right)\right]^{\mathrm{T}}$, $\partial \mu_{i j}^{k} / \partial \mathbf{x}_{i}=\left[\cos \left(\phi_{i j}\right)-\cos \left(\phi_{i k}\right) \sin \left(\phi_{i j}\right)-\sin \left(\phi_{i k}\right)\right]^{\mathrm{T}}$, $\partial \mu_{i j}^{k} / \partial \mathbf{x}_{k}=\left[\cos \left(\phi_{k j}\right)-\cos \left(\phi_{k i}\right) \sin \left(\phi_{k j}\right)-\sin \left(\phi_{k i}\right)\right]^{\mathrm{T}}$, and $\partial \mu_{i j}^{k} / \partial \mathbf{x}_{j}=\left[\cos \left(\phi_{j i}\right)+\cos \left(\phi_{j k}\right) \sin \left(\phi_{j i}\right)+\sin \left(\phi_{j k}\right)\right]^{\mathrm{T}}$, where $\phi_{i j}$ represents the angle between node $i$ and node $j$ with respect to to the horizontal axis (see Fig. 1). The entries in $\boldsymbol{\Sigma}^{-1}$ are also readily computed since $\boldsymbol{\Sigma}$ is block-diagonal so $\boldsymbol{\Sigma}^{-1}$ is block-diagonal as well (see Appendix A for additional details). Thus $\nabla_{\mathbf{x}} \boldsymbol{\mu}^{\mathrm{T}} \boldsymbol{\Sigma}^{-1} \nabla_{\mathrm{x}}^{\mathrm{T}} \boldsymbol{\mu}$ in (7) can be determined efficiently, even for large networks. Finally, the expectation over $\mathrm{x}$ in (7) can be performed through Monte Carlo integration.

Once the FIM is computed, the EFIM, and the PEB for individual nodes as well as for the entire network can be determined numerically.

\section{Bounds on Minimum MAC Delay}

Each TW-TOA transaction must be scheduled such that both nodes involved in the transaction are free from primary and secondary interference. Primary interference refers to a node not being able to transmit and receive at the same time, while secondary interference refers to a node not being able to receive multiple transmissions at the same time. This can be cast as a coloring problem on a suitable communication graph [34]. In [1], we have constructed tight lower and upper bounds on the minimum MAC delay, based on graphtheoretic arguments, in a complexity that is at most quadratic 
in the number of nodes. Due to space limitations, a detailed description is omitted here, and the reader is instead referred to [1], [2].

\section{SCALING LAWS}

While the numerical PEB and minimum MAC delay are useful to analyze particular networks, further insight can be gleaned from their asymptotic behavior, as the number of agents and anchors increases. The resulting scaling laws give fundamental understanding into the benefit and drawbacks of cooperation in UWB positioning, and simultaneously allow us to analyze methods to reduce the impact of the MAC delay, such as eavesdropping.

For reasons of tractability, we focus on dense networks [14], where every node can range with any other node, and wherein the area remains fixed and the node density increases by adding more nodes into the network. Moreover, we assume the ranging error variance to be constant for all transactions, as motivated in Section II.B. A general expression for the FIM under these assumptions is provided in Appendix B, where for notational and mathematical convenience we assume that anchors can also initiate TW-TOA procedures with other nodes. Scaling laws are derived from the EFIM in Appendices $\mathrm{C}-\mathrm{H}$. We recall that the PEB is expressed in meters, while the minimum MAC delay has a unit of seconds.

\section{A. Operating Conditions}

We analyze the scaling behavior of the PEB and minimum MAC delay for six distinct operating conditions: (i) noncooperative $(\mathrm{No})$, where agents perform TW-TOA with all anchors in communication range (i.e., $\mathcal{S}_{i}=\mathcal{N}_{i} \cap \mathcal{S}_{\text {anchors }}$ ); (ii) cooperative $(\mathrm{Co})$, where agents perform TW-TOA with all nodes in communication range (i.e., $\mathcal{S}_{i}=\mathcal{N}_{i}$ ); (iii) noncooperative with anchors eavesdropping (No-Ea), where agents perform TWTOA with all anchors while other anchors are able to perform eavesdropping measurements; (iv) noncooperative with all nodes eavesdropping (No-E), where agents perform TW-TOA with all anchors and all neighboring nodes are allowed to eavesdrop; (v) cooperative with anchors eavesdropping ( $\mathrm{Co}$ $E a$ ), where agents perform TW-TOA with all nodes in communication range and anchors are able to eavesdrop; and (vi) cooperative with eavesdropping $(\mathrm{Co}-\mathrm{E})$, where agents perform TW-TOA with all nodes in communication range and all nodes are able to eavesdrop.

Theorem 1. For a dense network, where measurements are given by (1)-(2), with a constant TOA variance, the PEB $(\mathcal{P})$, and the $M A C$ delay $(\mathcal{M})$ for the six operating conditions scale as listed in Table I.

Proof: See Appendices C-H.

Remarks: As was already noted in [14], in terms of the asymptotic PEB, agents play the same roles as anchors in the Co case. This causes the PEB to go down rapidly with the total number of nodes. However, we see that the MAC delay scaling also treats anchors as agents, thus causing a quadratic scaling in terms of the number of agents. This is the main reason why indiscriminate cooperation is prohibitive

\begin{tabular}{|c|c|c|}
\hline Scenario & $\mathcal{P} \in$ & $\mathcal{M} \in$ \\
\hline No & $\mathcal{O}\left(M^{-1 / 2}\right)$ & $\mathcal{O}(M N)$ \\
\hline Co & $\mathcal{O}\left((M+N)^{-1 / 2}\right)$ & $\mathcal{O}\left(M N+N^{2}\right)$ \\
\hline No-Ea & $\mathcal{O}\left(M^{-1}\right)$ & $\mathcal{O}(M N)$ \\
\hline No-E & $\mathcal{O}\left(\left(3 M^{2}+M N\right)^{-1 / 2}\right)$ & $\mathcal{O}\left(M N+M^{2}\right)$ \\
\hline Co-Ea & $\mathcal{O}\left(\left(M^{2}+M N\right)^{-1 / 2}\right)$ & $\mathcal{O}\left(N M+N^{2}\right)$ \\
\hline Co-E & $\mathcal{O}\left((M+N)^{-1}\right)$ & $\mathcal{O}\left((M+N)^{2}\right)$ \\
\hline
\end{tabular}

in terms of delay. The noncooperative case with only anchors eavesdropping (No-Ea) shows how the PEB is improved further, just by letting neighboring anchors listen to the TWTOA ranging procedures. The MAC delay scaling is the same as in the noncooperative scenario, as no additional TDMA slots are required to enable eavesdropping. Letting not only the anchors but also the agents eavesdrop (No-E) results in further enhancements in terms of PEB reduction. There is, however, an additional penalty in terms of delay, as anchors are allowed to range with each other, while agents eavesdrop. We clearly see the asymmetric role of agents and anchors in this scenario. The Co-Ea case, compared to standard cooperation, yields additional gains in terms of PEB. Note that in terms of the MAC delay, since there are no anchor-to-anchor transactions, but agent-to-agent transactions, the term $M^{2}$ present in the $N o-E$ case is replaced by $N^{2}$ in the Co-Ea case. In the last scenario $(C o-E)$, an order of magnitude reduction in PEB can be achieved by an order of magnitude increase in the number of nodes (agent or anchors), and two orders of magnitude increase in terms of the MAC delay. The role of agents and anchors is again symmetric.

\section{B. Trade-off Analysis}

The scaling laws above depend on the rate at which the number of agents $N$ increases with respect to the number of anchors $M$. To provide a unified view of the trade-off of the above scenarios, we will model $N=\kappa M^{\rho}$ to capture the growth of the number of agents relative to the number of anchors, and introduce the notion of the delay/accuracy tradeoff parameter $\delta(\rho) \in \mathbb{R}$, where $\rho$ is called the relative agent growth rate and $\kappa>0$. A similar tradeoff analysis concept can be found in [35] for a different context.

Definition 2 (Delay/accuracy trade-off parameter). Let $N=$ $\kappa M^{\rho}$, so that the PEB scales as $\mathcal{P} \in \mathcal{O}\left(f_{\mathcal{P}}(M, \rho)\right)$, while the MAC delay scales as $\mathcal{M} \in \mathcal{O}\left(f_{\mathcal{M}}(M, \rho)\right)$. The delay/accuracy trade-off is determined by

$$
\delta(\rho)=-\lim _{M \rightarrow+\infty} \frac{\log f_{\mathcal{P}}(M, \rho)}{\log f_{\mathcal{M}}(M, \rho)} .
$$

The trade-off parameter can be interpreted as the slope of the accuracy versus delay line in a log-log scale. Hence, operating conditions with a larger $\delta(\rho)$ will lead to a faster reduction in PEB as the delay increases than operating condi- 


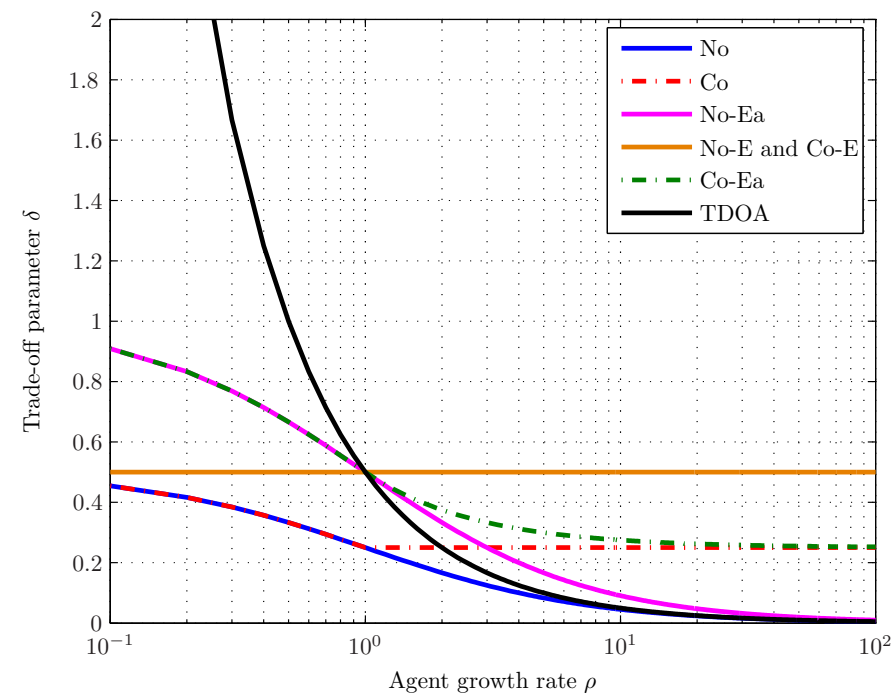

Figure 2. Delay/accuracy trade-off parameter $\delta$ as a function of the agent growth rate $\rho$ (i.e., $N \propto M^{\rho}$ ) for the different operating conditions.

tions with a smaller $\delta(\rho)$. In Fig. 2, we visualize the trade-off parameters for the six operating conditions as a function of the agent growth rate (note that the value of $\kappa$ is irrelevant). Additionally, for the sake of completeness and comparison purposes, the trade-off analysis for a time-difference-of-arrival (TDOA) scenario with $N$ agents and $M$ synchronized anchors is included. For this specific case the MAC delay scales as $\mathcal{M}_{\text {TDOA }} \in \mathcal{O}(N)$ and the PEB as $\mathcal{P}_{\text {TDOA }} \in \mathcal{O}(1 / \sqrt{M})$, which can be easily derived from [36, Equation (21)].

We observe that when $\rho<1$, so when anchors are added faster than agents, the best trade-off is achieved for TDOA followed by the $\mathrm{Co}-\mathrm{Ea}$ and $\mathrm{No}-\mathrm{Ea}$ cases. Interestingly, cooperation does not affect the trade-off (for example, No has exactly the same trade-off as Co), as the gain in PEB is canceled out by the increase in delay. For $\rho>1$, when agents are added faster than anchors, the situation changes: cooperative methods exhibit a better trade-off than their non-cooperative counterparts including TDOA. For very large growth rates, $T D O A$, No, and No-Ea, have a $\delta$ that tends to zero, meaning that there is almost no gain in terms of PEB when adding a few anchors and many agents. Similarly, $\mathrm{Co}$ and $\mathrm{Co}-\mathrm{Ea}$ converge to $\delta=1 / 4$ for large values of $\rho$, since the few additional anchors that eavesdrop do not significantly affect the PEB or delay. The value of $\delta=1 / 4$ should be interpreted as $\mathcal{M} \approx 1 / \mathcal{P}^{4}$, so that a $50 \%$ reduction in PEB leads to a 16 -fold increase in MAC delay. For $\rho>1$, the best trade-off is offered by the $N o-E$ and $C o-E$ cases. Interestingly, standard cooperation never offers the best trade-off.

\section{Numerical Results AND Discussion}

In this section, we go back to a non-asymptotic regime and evaluate the PEB and the upper and lower bounds on the MAC delay for four of the five operating conditions from Section IV. We analyze the impact of the number of anchors, the number of agents, the communication range, and cooperation among nodes. For each of the operating conditions, we also consider a selective variant where $\mathcal{S}_{i}$ can be a strict subset of $\mathcal{N}_{i}$.

\section{A. Simulation Setup}

We consider a $20 \mathrm{~m} \times 20 \mathrm{~m}$ square area: anchors are placed according to a scaled network topology from [13, Fig. 13] while agents are uniformly distributed in the fixed area. Based on our experimental results with the P400 UWB radios [28], we consider a ranging standard deviation ${ }^{2}$ of 2 $\mathrm{cm}$ (irrespective of distance under line-of-sight propagation, as argued in Section II.B) and TDMA time slot duration of $20 \mathrm{~ms}$. The a priori distributions of the agents' positions are Gaussian with unit variance. In addition to the scenarios $N o$, Co, No-E, Co-E, we also introduce selective ranging, leading to additional operation conditions: Noncooperative Selective $(\mathrm{No}-\mathrm{S})$ and Noncooperative Eavesdropping Selective (No-ES) (where $\mathcal{S}_{i} \subseteq \mathcal{N}_{i} \cap \mathcal{S}_{\text {anchors, }}$, such that $\left|\mathcal{S}_{i}\right| \leq 4$ ), and Cooperative Selective $(\mathrm{Co}-\mathrm{S})$ and Cooperative Eavesdropping Selective $(C o-E-S)$ (where $\mathcal{S}_{i} \subseteq \mathcal{N}_{i}$, such that $\left|\mathcal{S}_{i}\right| \leq 4$ ). The selection of the nodes in $\mathcal{S}_{i}$ is implemented using a distributed greedy algorithm to minimize the local PEB, described in [2].

\section{B. Impact of Number of Anchors}

The impact on the localization accuracy and MAC delay for a complete network graph with 10 agents and an increasing number of anchors (from 2 to 10) is illustrated in Fig. 3. The upper and lower bounds for the MAC delay for a clique network are the same, thus Fig. 3 only depicts the lower bounds. Each curve in the figure contains 9 markers, each marker corresponds to the increasing number of anchors $M$ (2 to 10) from left to right. All scenarios show a decrease in the PEB when increasing the number of anchors as validated in previous works. However, the improvement in accuracy comes with a cost in delay, which is linear in $M$. From the figure, it is also clear that cooperation $(\mathrm{Co})$ exhibits a poor delay/accuracy tradeoff. For example, consider a network with 5 anchors, for the No case the PEB is $\approx 1.3 \mathrm{~cm}$, while for $C o$ the PEB is $\approx 0.7 \mathrm{~cm}$. However, the accuracy comes with a cost in delay to the amount of $\approx 1 \mathrm{~s}$ for $\mathrm{No}$ and $\approx 3.3 \mathrm{~s}$ for $\mathrm{Co}$.

For the eavesdropping cases (No-E and Co-E), we observe an improvement with respect to the non-eavesdropping cases ( $\mathrm{No}$ and $\mathrm{Co}$ ) without incurring any extra delay since the eavesdropping measurements do not require any scheduling. Hence all corresponding curves shift downward. We can observe that the results are in accordance with the scaling laws, where the reduction of the PEB and the increase in MAC delay are dependent on the number of anchors, with more PEB reduction for the eavesdropping cases while maintaining the same MAC delay similarly to the non-eavesdropping cases.

When selective ranging is employed, we see that for $\mathrm{No}$ $S$ the PEB no longer improves for $M \geq 4$ and the MAC delay stays constant just below $1 \mathrm{~s}$. Thus, Fig. 3 only shows 3 distinct markers for $\mathrm{No}-\mathrm{S}$. The $\mathrm{Co}-\mathrm{S}$ case corresponds to closely placed markers in Fig. 3, since the agents can always find enough neighbors (agents or anchors) to cooperate with. The No-E-S and Co-E-S cases result in a reduction of the PEB without any additional MAC delay. The advantage of adding anchors is clearer for $C o-E-S$ than for $C o-S$.

\footnotetext{
${ }^{2}$ The results we will present can be adapted to any other ranging standard deviation through proper scaling of the PEB.
} 


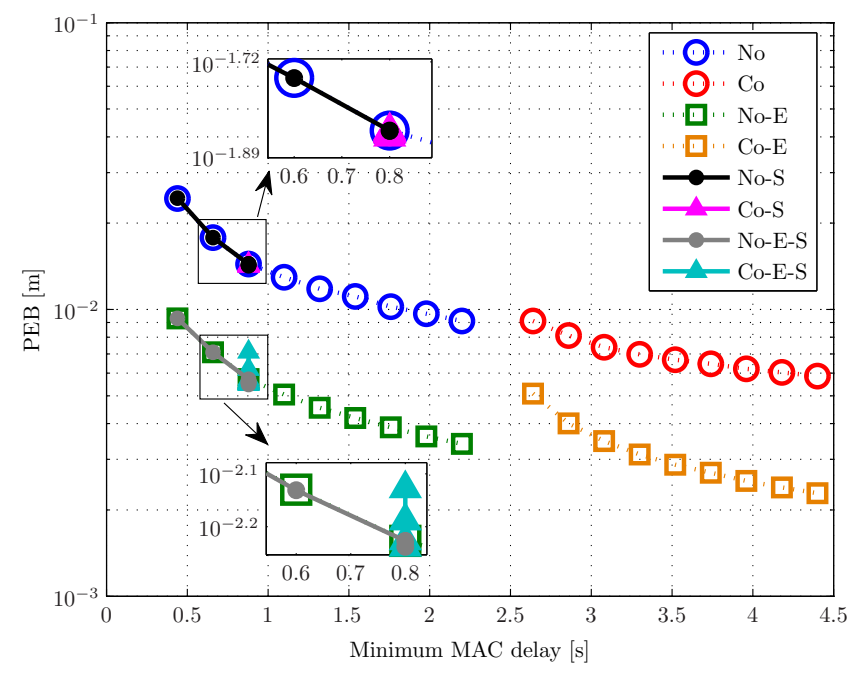

Figure 3. MAC delay and PEB lower bounds for a clique network with 10 agents and increasing number of anchors (2 to 10). Each curve consists of 9 markers, each marker representing the increase in the number of anchors $M$ from left to right, except for the selective cases.

\section{Impact of Number of Agents}

Fig. 4 depicts the impact on the localization accuracy and the MAC delay for a complete network graph with 3 anchors and an increasing number of agents (from 1 to 20). Each curve in the figure consists of 20 markers, each one corresponding to the increasing number of agents $N$ (1 to 20) from left to right. Note that the $\mathrm{No}, \mathrm{No}-\mathrm{S}$, and $\mathrm{Co}-\mathrm{S}$ cases coincide and therefore are grouped into one single label: Group 1. Similarly, the No$E, N o-E-S$, and $C o-E-S$ are grouped into the label: Group 2. Once again, the lower and upper bounds on the MAC delay are the same, and only the lower bound is presented in Fig. 4.

The addition of agents to the network has no impact in the PEB for Group 1, but increases the MAC delay since more agent-to-anchor TW-TOA procedures need to be scheduled. For Group 2, adding agents to the network translates to a rapid decrease in the $\mathrm{PEB}$, since for this particular group adding agents means more information, as agents are able to eavesdrop TW-TOA ranging transactions between agents and anchors. For the Co case, adding more agents decreases the PEB as compared with Group 1, since more agent-to-agent TW-TOA information is available in the network. However, cooperation comes with a cost in delay which grows quadratic in $N$. The $C o$ PEB can be reduced without any additional MAC delay by eavesdropping, leading to the $C o-E$ curve.

We conclude that full cooperation with many agents is not feasible when there are tight delay constraints, for example in the case of highly dynamic agents. Hence, theoretical cooperative gains cannot be exploited. We can observe the importance of the number of agents especially for the cooperative cases, with the corresponding cost in delay and PEB reduction consistent with the scaling laws.

\section{Impact of Communication Range}

For the analysis of the communication range variable we consider a network consisting of 20 agents and 13 anchors, and an increasing communication range $R$ from $1 \mathrm{~m}$ to $30 \mathrm{~m}$. Each

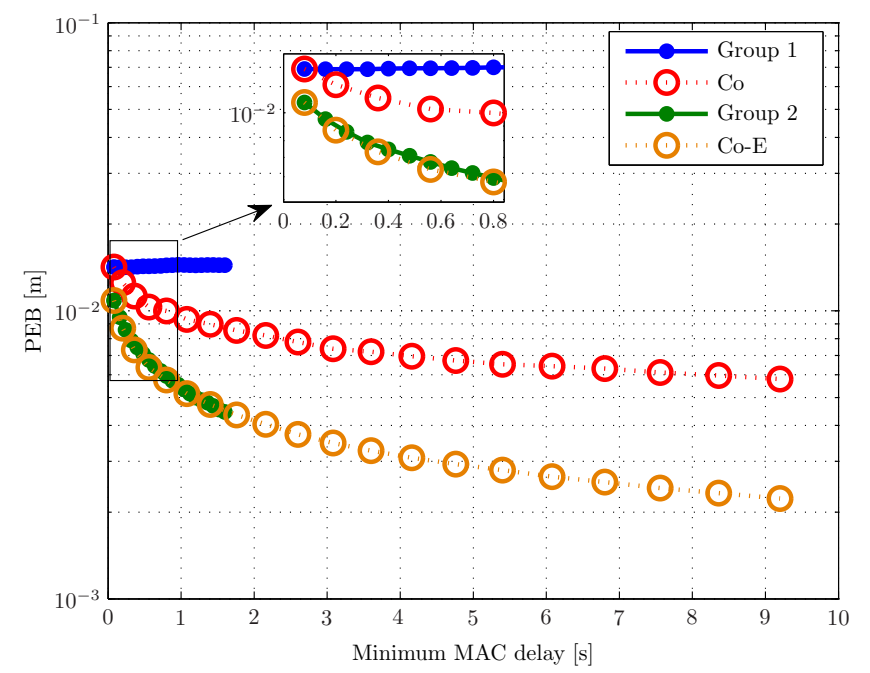

Figure 4. MAC delay and PEB lower bounds for a clique network with 3 anchors and increasing number of agents (1 to 20). Each curve consists of 20 markers, each marker representing the increase in the number of agents $N$ from left to right. Group 1 consists the scenarios: No, No-S, and $\mathrm{Co}-\mathrm{S}$. Group 2 contains the cases: No-E, No-E-S, and Co-E-S.

marker in the curves represents the nominal communication range $R$ used in the communication model (see Section II.C) increasing from left to right, i.e., the leftmost and rightmost markers of each curve represent $R=1 \mathrm{~m}$, and $R=30 \mathrm{~m}$, respectively. Figs. 5 and 6 show the influence in the PEB and MAC delay trade-off for the noncooperative cases $(\mathrm{No}$, $\mathrm{No}-\mathrm{S}, \mathrm{No}-\mathrm{E}, \mathrm{No}-\mathrm{E}-\mathrm{S})$ and the cooperative cases (Co, Co-S, $C o-E$, and $C o-E-S)$, respectively. For low values of $R$, the lower and upper bounds on the minimum MAC delay do not coincide, though the bounds are relatively tight for all $R$ under consideration.

In Fig. 5, as expected, increasing the communication range decreases the PEB since more agent-to-anchor transactions are injected into the network, though once again with a cost in delay. For the No-S case, the curve remains constant in both accuracy and delay once agents are able to communicate with at least four anchors (this happens when $R$ is around $6 \mathrm{~m}$ ). In contrast, even though $N o-E-S$ remains constant once agents can communicate with at least four nodes, increasing range allows for more nodes in the network to perform eavesdropping, hence the PEB improvement for this specific case results in vertical drop on PEB. This clearly shows that using high-power anchors is only meaningful with a selective ranging strategy. The No-E scenario outperforms $N o$, once again with a cost in delay.

In Fig. 6 we observe that cooperative selective cases show a similar behavior as the noncooperative counterpart, though with more extreme values in terms of PEB and minimum MAC delay. The PEB decreases with increasing $R$ while the MAC delay grows fast reaching up to $N \times M \times 20 \mathrm{~ms} \approx 5 \mathrm{~s}$, and $N \times(M+(N-1)) \times 20 \mathrm{~ms} \approx 13 \mathrm{~s}$ for $N o$ and $C o$, respectively. Evidently, the latter shows that increasing $R$ for a marginal gain in terms of accuracy can lead to large delays, especially when cooperation is implemented indiscriminately. 


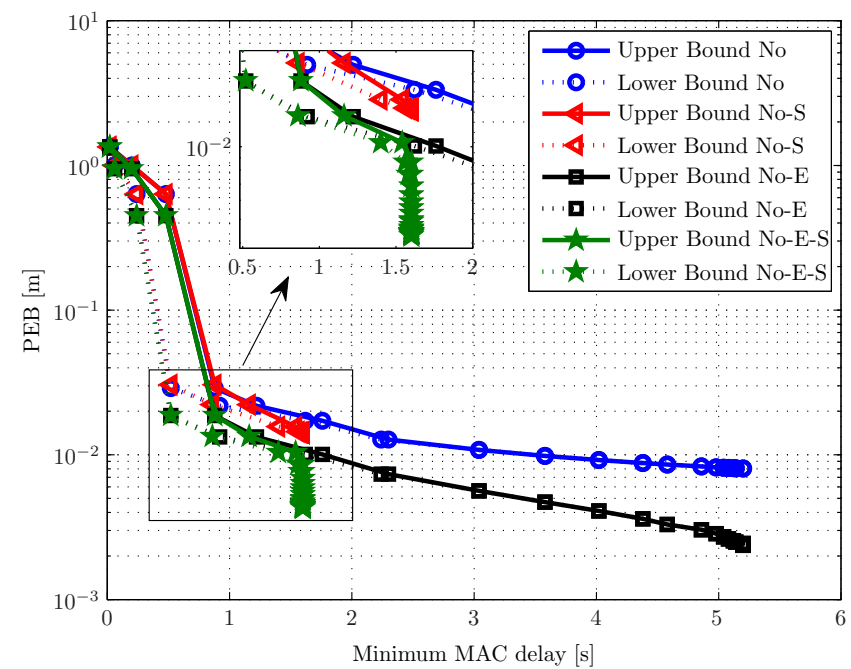

Figure 5. MAC delay and PEB lower bounds for a partially noncooperative connected network with 20 anchors and 13 agents. Each curve consists of 30 markers, each marker representing the increase in the nominal communication range $R$ from left to right (1 to $30 \mathrm{~m}$ ).

\section{CONCLUSIONS}

We have investigated the interplay between UWB positioning accuracy and MAC delay. We presented lower bounds on the position accuracy and the MAC delay considering spatial time division multiple access for arbitrary finite networks. We have characterized the behavior for dense-location aware networks for the noncooperative and cooperative cases by means of the relevant scaling laws. We found that traditional methods to improve accuracy, such as increasing the number of anchors or the communication range, or the implementation of cooperation among nodes comes at a cost in terms of MAC delay. The latter has a direct impact in the update rate when dealing with dynamic networks with respect to mobility. Selective ranging and eavesdropping have been evaluated as possible methods to reduce the MAC delay with reasonable position accuracy. Noncooperative eavesdropping shows to outperform cooperative networks in terms of accuracy with reasonable delays. Finally, in terms of scaling, we found that, under certain conditions, standard cooperative positioning exhibits the worst possible trade-off among the considered strategies.

Possible avenues of future research include the extension of the scaling laws to non-complete networks, to different MAC protocols and measurement aggregation techniques, differences in measurement variance dependent on distance, and to network problems outside of positioning.

\section{APPENDIX A \\ Structure of THE INVERSE COVARIANCE MATRIX}

We consider a network with agents and anchors and focus on a particular agent $i$ with a neighbor $j$ and a collection of $U-1$ eavesdroppers $k \in \mathcal{N}_{i} \cap \mathcal{N}_{j}$. The resulting measurement is $\mathbf{z}_{i j}$, as defined in Section II.B. The corresponding covariance

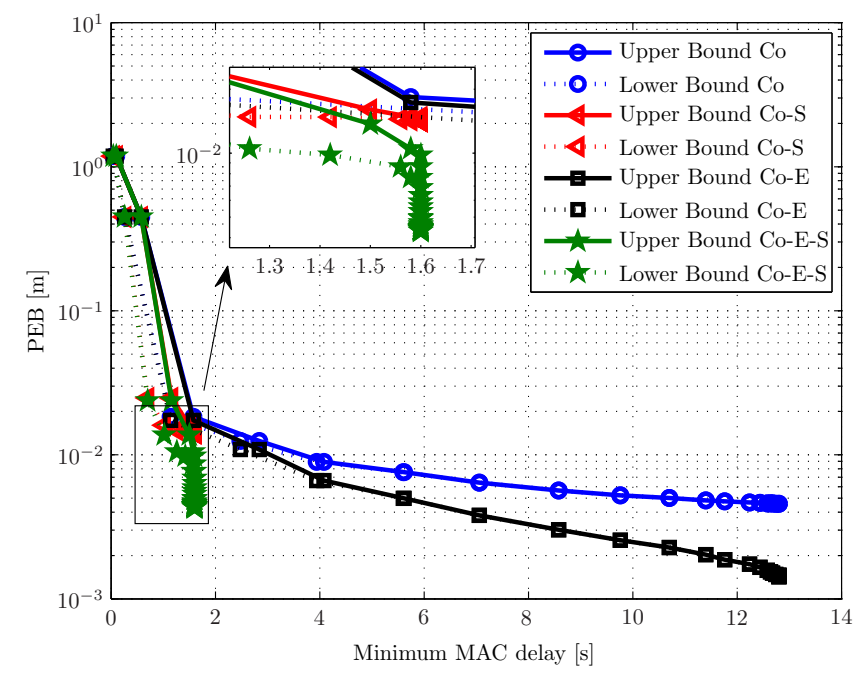

Figure 6. MAC delay and PEB lower bounds for a partially cooperative connected network with 20 anchors and 13 agents. Each curve consists of 30 markers, each marker representing the increase in the nominal communication range $R$ from left to right ( 1 to $30 \mathrm{~m}$ ).

matrix $\mathbf{C}_{i j}$ for constant ranging error variance is given by

$$
\mathbf{C}_{i j}=\sigma^{2}\left[\begin{array}{ccccc}
1 / 2 & 1 / 2 & 1 / 2 & \ldots & 1 / 2 \\
1 / 2 & 3 & 1 & \ldots & 1 \\
1 / 2 & 1 & 3 & \ldots & 1 \\
\vdots & \vdots & \vdots & \ddots & \vdots \\
1 / 2 & 1 & 1 & \cdots & 3
\end{array}\right]
$$

It is readily verified that the inverse is given by

$$
\mathbf{C}_{i j}^{-1}=\frac{1}{\sigma^{2}}\left[\begin{array}{ccccc}
\alpha & \gamma & \gamma & \cdots & \gamma \\
\gamma & \beta & \gamma & \cdots & \gamma \\
\gamma & \gamma & \beta & \cdots & \gamma \\
\vdots & \vdots & \vdots & \ddots & \vdots \\
\gamma & \gamma & \gamma & \cdots & \beta
\end{array}\right]
$$

where

$$
\begin{aligned}
\alpha & =2\left(1+\frac{U-1}{U+3}\right) \\
\beta & =\frac{U+2}{2(U+3)} ; \gamma=\frac{-2}{(U+3)} \\
\eta & =\frac{-1}{2(U+3)} .
\end{aligned}
$$

In the particular case where all anchors (except anchor $j$ ) eavesdrop, $U=M$, and in the particular case where all nodes (except node $j$ ) eavesdrop, $U=M+N-1$. In the special case when there are no eavesdroppers, $\mathbf{C}_{i j}^{-1}$ reverts to the scalar value 2 (i.e., $\beta=\gamma=\eta=0$, and $\alpha=2$ ).

\section{APPENDIX B}

\section{General Form OF THE Classical FIM}

In this section we introduce the most generalized form of the classical FIM, denoted here by $\mathbf{J}$ (not to be confused with the Bayesian FIM in (7)). For convenience of the notation, we will assume that anchors can also initiate TW-TOA procedures with other nodes. We also omit the ranging variance $\sigma^{2}$, with 
the understanding that the FIM is to be multiplied by $1 / \sigma^{2}$. Let $\mathcal{S}_{R}$ be the set of all nodes with which agents can range and $\mathcal{S}_{E}$ the set of nodes that perform eavesdropping. We introduce $\left[\gamma_{*}, \beta_{*}, \eta_{*}\right]$, which are set to zero when only anchors eavesdrop, and set to $[\gamma, \beta, \eta]$ when both anchors and agents eavesdrop. When no-one eavesdrops, $\left[\gamma_{*}, \beta_{*}, \eta_{*}\right]=[\gamma, \beta, \eta]=$ $[0,0,0]$. The diagonal block-elements of the FIM are given by $[\mathbf{J}]_{k k}$

$$
\begin{aligned}
& =2 \alpha \sum_{j \in \mathcal{S}_{R} \backslash\{k\}}\left[\left(\frac{\partial \mu_{k j}}{\partial \mathbf{x}_{k}}\right)\left(\frac{\partial \mu_{k j}}{\partial \mathbf{x}_{k}}\right)^{\mathrm{T}}\right] \\
& +4 \gamma \sum_{j \in \mathcal{S}_{R} \backslash\{k\}} \sum_{n \in \mathcal{S}_{E} \backslash\{j, k\}}\left[\left(\frac{\partial \mu_{k j}}{\partial \mathbf{x}_{k}}\right)\left(\frac{\partial \mu_{k j}^{n}}{\partial \mathbf{x}_{k}}\right)^{\mathrm{T}}\right] \\
& +2 \eta \sum_{j \in \mathcal{S}_{R} \backslash\{k\}} \sum_{n \in \mathcal{S}_{E} \backslash\{j, k\}} \sum_{m \in \mathcal{S}_{E} \backslash\{j, k, n\}}\left[\left(\frac{\partial \mu_{k j}^{m}}{\partial \mathbf{x}_{k}}\right)\left(\frac{\partial \mu_{k j}^{n}}{\partial \mathbf{x}_{k}}\right)^{\mathrm{T}}\right]
\end{aligned}
$$

$$
\begin{aligned}
& +2 \beta_{*} \sum_{j \in \mathcal{S}_{R} \backslash\{k\}} \sum_{m \in \mathcal{S}_{R} \backslash\{j, k\}}\left[\left(\frac{\partial \mu_{m j}^{k}}{\partial \mathbf{x}_{k}}\right)\left(\frac{\partial \mu_{m j}^{k}}{\partial \mathbf{x}_{k}}\right)^{\mathrm{T}}\right] \\
& +2 \beta \sum_{j \in \mathcal{S}_{R} \backslash\{k\}} \sum_{n \in \mathcal{S}_{E} \backslash\{j, k\}}\left[\left(\frac{\partial \mu_{k j}^{n}}{\partial \mathbf{x}_{k}}\right)\left(\frac{\partial \mu_{k j}^{n}}{\partial \mathbf{x}_{k}}\right)^{\mathrm{T}}\right] .
\end{aligned}
$$

Each term has a corresponding interpretation: for example (14b) corresponds to the information regarding the position of agent $k$, due to correlation between the TW-TOA measurement from the ranging transaction initiated by agent $k$ to node $j$, and the eavesdropping measurement by node $n$ with respect to that same ranging transaction.

For $k \neq l$, we have even more combinations:

$[\mathbf{J}]_{k l}$

$$
=2 \alpha\left(\frac{\partial \mu_{k l}}{\partial \mathbf{x}_{k}}\right)\left(\frac{\partial \mu_{k l}}{\partial \mathbf{x}_{l}}\right)^{\mathrm{T}}
$$$$
+2 \gamma \sum_{n \in \mathcal{S}_{E}}\left(\frac{\partial \mu_{k l}}{\partial \mathbf{x}_{k}}\right)\left(\frac{\partial \mu_{k l}^{n}}{\partial \mathbf{x}_{l}}\right)^{\mathrm{T}}+\left(\frac{\partial \mu_{k l}^{n}}{\partial \mathbf{x}_{k}}\right)\left(\frac{\partial \mu_{k l}}{\partial \mathbf{x}_{l}}\right)^{\mathrm{T}}
$$$$
+\gamma_{*} \sum_{n \in \mathcal{S}_{R}}\left(\frac{\partial \mu_{k n}}{\partial \mathbf{x}_{k}}\right)\left(\frac{\partial \mu_{k n}^{l}}{\partial \mathbf{x}_{l}}\right)^{\mathrm{T}}+\left(\frac{\partial \mu_{l n}^{k}}{\partial \mathbf{x}_{k}}\right)\left(\frac{\partial \mu_{l n}}{\partial \mathbf{x}_{l}}\right)^{\mathrm{T}}
$$$$
+\beta \sum_{m \in \mathcal{S}_{E}}\left(\frac{\partial \mu_{k l}^{m}}{\partial \mathbf{x}_{k}}\right)\left(\frac{\partial \mu_{k l}^{m}}{\partial \mathbf{x}_{l}}\right)^{\mathrm{T}}+\left(\frac{\partial \mu_{l k}^{m}}{\partial \mathbf{x}_{k}}\right)\left(\frac{\partial \mu_{l k}^{m}}{\partial \mathbf{x}_{l}}\right)^{\mathrm{T}}
$$

$$
+\beta_{*} \sum_{n \in \mathcal{S}_{R}}\left(\frac{\partial \mu_{k n}^{l}}{\partial \mathbf{x}_{k}}\right)\left(\frac{\partial \mu_{k n}^{l}}{\partial \mathbf{x}_{l}}\right)^{\mathrm{T}}+\left(\frac{\partial \mu_{l n}^{k}}{\partial \mathbf{x}_{k}}\right)\left(\frac{\partial \mu_{l n}^{k}}{\partial \mathbf{x}_{l}}\right)^{\mathrm{T}}
$$$$
+\beta_{*} \sum_{n \in \mathcal{S}_{R}}\left(\frac{\partial \mu_{n k}^{l}}{\partial \mathbf{x}_{k}}\right)\left(\frac{\partial \mu_{n k}^{l}}{\partial \mathbf{x}_{l}}\right)^{\mathrm{T}}+\left(\frac{\partial \mu_{n l}^{k}}{\partial \mathbf{x}_{k}}\right)\left(\frac{\partial \mu_{n l}^{k}}{\partial \mathbf{x}_{l}}\right)^{\mathrm{T}}
$$

$+\eta \sum_{m \in \mathcal{S}_{E}} \sum_{n \in \mathcal{S}_{E}}\left(\frac{\partial \mu_{k l}^{n}}{\partial \mathbf{x}_{k}}\right)\left(\frac{\partial \mu_{k l}^{m}}{\partial \mathbf{x}_{l}}\right)^{\mathrm{T}}+\left(\frac{\partial \mu_{l k}^{n}}{\partial \mathbf{x}_{k}}\right)\left(\frac{\partial \mu_{l k}^{m}}{\partial \mathbf{x}_{l}}\right)^{\mathrm{T}}$

\begin{tabular}{|c|c|}
\hline Term & Scaling \\
\hline \hline (14a) in $[\mathbf{J}]_{k k}$ & $2 \alpha N_{R} \mathbf{I}_{2}$ \\
\hline$(14 \mathrm{~b})$ in $[\mathbf{J}]_{k k}$ & $2 \gamma N_{E} N_{R} \mathbf{I}_{2}$ \\
\hline (14c) in $[\mathbf{J}]_{k k}$ & $\eta N_{R} N_{E}^{2} \mathbf{I}_{2}$ \\
\hline (14d) in $[\mathbf{J}]_{k k}$ & $2 \beta_{*} N_{R}^{2} \mathbf{I}_{2}$ \\
\hline (14e) in $[\mathbf{J}]_{k k}$ & $2 \beta N_{R} N_{E} \mathbf{I}_{2}$ \\
\hline (15a) in $[\mathbf{J}]_{k l}$ & $-2 \alpha \mathbf{A}\left(\phi_{k l}\right)$ \\
\hline (15b) in $[\mathbf{J}]_{k l}$ & $-4 \gamma N_{E} \mathbf{A}\left(\phi_{k l}\right)$ \\
\hline$(15 \mathrm{c}),(15 \mathrm{~h}),(15 \mathrm{i}),(15 \mathrm{j})$ in $[\mathbf{J}]_{k l}$ & $\mathbf{0}$ \\
\hline (15d) in $[\mathbf{J}]_{k l}$ & $-2 \beta N_{E} \mathbf{A}\left(\phi_{k l}\right)$ \\
\hline (15d) in $[\mathbf{J}]_{k l}$ & $-2 \beta_{*} N_{R} \mathbf{A}\left(\phi_{k l}\right)$ \\
\hline$(15 \mathrm{f})$ in $[\mathbf{J}]_{k l}$ & $-2 \beta_{*} N_{R} \mathbf{A}\left(\phi_{k l}\right)$ \\
\hline (15g) in $[\mathbf{J}]_{k l}$ & $-2 \eta N_{E}^{2} \mathbf{A}\left(\phi_{k l}\right)$ \\
\hline
\end{tabular}

Table II

SCALING OF THE DIFFERENT TERMS IN $[\mathbf{J}]_{k k}$ AND $[\mathbf{J}]_{k l}$.

$+\eta_{*} \sum_{m \in \mathcal{S}_{E}} \sum_{n \in \mathcal{S}_{R}}\left(\frac{\partial \mu_{k n}^{m}}{\partial \mathbf{x}_{k}}\right)\left(\frac{\partial \mu_{k n}^{l}}{\partial \mathbf{x}_{l}}\right)^{\mathrm{T}}+\left(\frac{\partial \mu_{n k}^{m}}{\partial \mathbf{x}_{k}}\right)\left(\frac{\partial \mu_{n k}^{l}}{\partial \mathbf{x}_{l}}\right)^{\mathrm{T}}$

$+\eta_{*} \sum_{m \in \mathcal{S}_{E}} \sum_{n \in \mathcal{S}_{R}}\left(\frac{\partial \mu_{l n}^{k}}{\partial \mathbf{x}_{k}}\right)\left(\frac{\partial \mu_{l n}^{m}}{\partial \mathbf{x}_{l}}\right)^{\mathrm{T}}+\left(\frac{\partial \mu_{n l}^{k}}{\partial \mathbf{x}_{k}}\right)\left(\frac{\partial \mu_{n l}^{m}}{\partial \mathbf{x}_{l}}\right)^{\mathrm{T}}$

$+\eta_{*} \sum_{n \in \mathcal{S}_{R}} \sum_{m \in \mathcal{S}_{R}}\left(\frac{\partial \mu_{n m}^{k}}{\partial \mathbf{x}_{k}}\right)\left(\frac{\partial \mu_{n m}^{l}}{\partial \mathbf{x}_{l}}\right)^{\mathrm{T}}$.

Denoting the cardinality of $\mathcal{S}_{R}$ (resp. $\mathcal{S}_{E}$ ) by $N_{R}$ (resp. $N_{E}$ ), and assuming the nodes are dropped uniformly around agents $k$ and $l$, we can use the expression for the partial derivatives from Section III.B to determine the scaling of each term in (14) and (15). The scaling of each term is show in Table II, where $\mathbf{I}_{2}$ stands for the $2 \times 2$ identity matrix, and where

$$
\mathbf{A}\left(\phi_{k l}\right)=\left[\begin{array}{cc}
\cos ^{2} \phi_{k l} & \cos \phi_{k l} \sin \phi_{k l} \\
\sin \phi_{k l} \cos \phi_{k l} & \sin ^{2} \phi_{k l}
\end{array}\right] .
$$

We recall that $\alpha, \gamma, \eta, \beta_{*}, \beta$ take on values that depend on $N_{E}$ and $N_{R}$, depending on the specific scenario.

\section{APPENDIX C Proof of the No Case}

\section{A. $P E B$}

Consider adding $M$ anchors and $N$ agents uniformly distributed over a fixed two-dimensional area. Moreover, no prior information is available for the agents' positions. We will determine the scaling of the equivalent Fisher information matrix (EFIM) $\mathbf{J}_{\text {No }}^{\mathrm{E}}$ for agent $k$. Since the TW-TOA transactions are mutually independent, the FIM is a block diagonal matrix of the form $\mathbf{J}_{\mathrm{No}}=\operatorname{diag}\left[\mathbf{J}_{\mathrm{No}, k}, \mathbf{J}_{\mathrm{No}, 2}, \ldots, \mathbf{J}_{\mathrm{No}, N}\right]$. Given the definition of the EFIM from Section III.A, for this specific case 
we have that $\mathbf{J}_{\mathrm{No}, k}^{\mathrm{E}}=\mathbf{J}_{\mathrm{No}, k}$. From the generalized expression in (14), we can construct $\mathbf{J}_{\text {No }}^{\mathrm{E}}$ by considering only the term (14a), with $\mathcal{S}_{R}=\mathcal{S}_{\text {anchors }}, N_{R}=M$. Therefore, from Table II the EFIM scales as $\mathbf{J}_{\mathrm{No}, k}^{\mathrm{E}}=\mathbf{J}_{\mathrm{No}, k} \rightarrow 2 \alpha N_{R} \mathbf{I}_{2}=4 M \mathbf{I}_{2}$, where the notation $A \rightarrow B$ depicts the scaling of expression $A$ in the asymptotic regime (large number of nodes) deriving in expression $B$. Finally, following the definition of the $\mathrm{PEB}$, we find that for large $M, \mathcal{P}_{\mathrm{No}} \rightarrow \sqrt{1 /(4 M)}$. Hence, $\mathcal{P}_{\text {No }} \in \mathcal{O}(1 / \sqrt{M})$.

\section{B. Minimum MAC Delay}

For a clique network with $N$ agents, each agent must range with $M$ anchors. Each transaction requires a separate TDMA time slot. Thus, all agent-to-anchor links need to be scheduled within the network, therefore the minimum MAC delay is exactly $\mathcal{M}_{\text {No }} \in \mathcal{O}(M N)$.

APPENDIX D

\section{Proof of the Co CASE}

\section{A. PEB}

When the $N$ agents cooperate, the FIM $\mathbf{J}_{\text {Co }}$ comprises $2 \times 2$ block matrices. From the generalized expressions in equations (14) and (15), the diagonal blocks $(k=l)\left[\mathbf{J}_{\mathrm{Co}}\right]_{k k}$ are computed by considering only the term (14a) while the non-diagonal blocks $(k \neq l)$ consist of the term (15a) with $\mathcal{S}_{R}=\mathcal{S}_{\text {anchors }} \cup \mathcal{S}_{\text {agents, }}$, with $\alpha=2$. Hence, following Table II, since $N_{R}=M+N-1$ and $\alpha=2$,

$$
\left[\mathbf{J}_{\mathrm{Co}}\right]_{k l} \rightarrow \begin{cases}4(M+N) \mathbf{I}_{2} & k=l \\ -4 \mathbf{A}\left(\phi_{k l}\right) & k \neq l .\end{cases}
$$

The EFIM itself is hard to compute, so we will follow the procedure from [14] and determine matrices $\mathbf{J}_{\mathrm{Co}}^{\mathrm{L}}$ and $\mathbf{J}_{\mathrm{Co}}^{\mathrm{U}}$ that satisfy $\mathbf{J}_{\mathrm{Co}}^{\mathrm{L}} \preceq \mathbf{J}_{\mathrm{Co}} \preceq \mathbf{J}_{\mathrm{Co}}^{\mathrm{U}}$, based on which we can determine an EFIM in closed form. Without loss of generality, we focus on agent $k=1$ and analyze the corresponding EFIM lower and upper bounds.

EFIM Lower Bound: We construct $\mathbf{J}_{\mathrm{Co}}^{\mathrm{L}}$ from $\mathbf{J}_{\mathrm{Co}}$ by removing all cooperation information except where agent 1 is involved in a ranging transaction, so that the diagonal elements of the FIM are given by $\left[\mathbf{J}_{\mathrm{Co}}^{\mathrm{L}}\right]_{11}=4(M+N) \mathbf{I}_{2}$ and, for $k \neq 1,\left[\mathbf{J}_{\mathrm{Co}}^{\mathrm{L}}\right]_{k k}=4(M+1) \mathbf{I}_{2}$. The off-diagonal elements are

$$
\left[\mathbf{J}_{\mathrm{Co}}^{\mathrm{L}}\right]_{k l}= \begin{cases}-4 \mathbf{A}\left(\phi_{k l}\right) & k=1, l>1 \\ -4 \mathbf{A}\left(\phi_{k l}\right) & l=1, k>1 \\ \mathbf{0} & k \neq l, k, l>1 .\end{cases}
$$

Since information is removed, it follows immediately that $\mathbf{J}_{\text {Co }}^{\mathrm{L}} \preceq \mathbf{J}_{\text {Co }}$. Using Schur's complement, the lower bound on the EFIM of agent 1 is now given by

$$
\mathbf{J}_{\mathrm{Co}, 1}^{\mathrm{L}, \mathrm{E}}=\left[\mathbf{J}_{\mathrm{Co}}^{\mathrm{L}}\right]_{11}-\sum_{k \in \mathcal{S}_{\text {agents }} \backslash\{1\}}\left[\mathbf{J}_{\mathrm{Co}}^{\mathrm{L}}\right]_{1 k}\left[\mathbf{J}_{\mathrm{Co}}^{\mathrm{L}}\right]_{k k}^{-1}\left[\mathbf{J}_{\mathrm{Co}}^{\mathrm{L}}\right]_{1 k}^{\mathrm{T}} \text {. }
$$

The second term scales as

$$
\sum_{k \in \mathcal{S}_{\text {agents } \backslash\{1\}}}\left[\mathbf{J}_{\mathrm{Co}}^{\mathrm{L}}\right]_{1 k}\left[\mathbf{J}_{\mathrm{Co}}^{\mathrm{L}}\right]_{k k}^{-1}\left[\mathbf{J}_{\mathrm{Co}}^{\mathrm{L}}\right]_{1 k}^{\mathrm{T}} \rightarrow \frac{N}{M} \mathbf{I}_{2} .
$$

Hence, for sufficiently large $M$ and $N, \mathbf{J}_{\mathrm{Co}, 1}^{\mathrm{L}, \mathrm{E}} \rightarrow 4(M+N) \mathbf{I}_{2}$.
EFIM Upper Bound: The EFIM upper bound $\mathbf{J}_{\mathrm{Co}}^{\mathrm{U}}$ is constructed from $\mathbf{J}_{\mathrm{Co}}$ by setting the non-diagonal block elements equal to zero, i.e., $\left[\mathbf{J}_{\mathrm{Co}}^{\mathrm{L}}\right]_{k l}=0$ for $k \neq l$. The EFIM for agent 1 is now $\mathbf{J}_{\mathrm{Co}, 1}^{\mathrm{U}, \mathrm{E}}=\left[\mathbf{J}_{\mathrm{Co}}\right]_{11}=4(M+N) \mathbf{I}_{2}$.

Final Scaling: Since the EFIM scales at least as fast as $4(M+N) \mathbf{I}_{2}$ and at most as fast as $4(M+N) \mathbf{I}_{2}$, we conclude that $\mathbf{J}_{\mathrm{Co}, 1}^{\mathrm{E}} \rightarrow 4(M+N) \mathbf{I}_{2}$. According to the definition of the PEB, we finally find that $\mathcal{P}_{\mathrm{Co}} \rightarrow 1 / \sqrt{4(M+N)}$, and thus $\mathcal{P}_{\text {Co }} \in \mathcal{O}(1 / \sqrt{M+N})$.

\section{B. Minimum MAC Delay}

For a cooperative clique network each of the $N$ agents performs a TW-TOA transaction with every one of the remaining $N+M-1$ nodes. Each transaction requires a different TDMA slot, so the total MAC delay scales as $\mathcal{M}_{\text {Co }} \in \mathcal{O}\left(N M+N^{2}\right)$.

\section{APPENDIX E}

Proof of The No-Ea CASE

\section{A. $P E B$}

Consider the addition of $M$ anchors and $N$ agents uniformly distributed over a fixed two-dimensional area. The FIM $\mathbf{J}_{\mathrm{No}-\mathrm{Ea}}$ in the case where nodes range only with anchors and only anchors eavesdrop is a block-diagonal matrix of the form $\mathbf{J}_{\mathrm{No}-\mathrm{Ea}}=\operatorname{diag}\left[\mathbf{J}_{\mathrm{No}-\mathrm{Ea}, 1}, \ldots, \mathbf{J}_{\mathrm{No}-\mathrm{Ea}, N}\right]$. Given the definition of the EFIM from Section III.A, for this case we have that $\mathbf{J}_{\mathrm{No}-\mathrm{Ea}, k}^{\mathrm{E}}=\mathbf{J}_{\mathrm{No}-\mathrm{Ea}, k}$. From the generalized expression (14) we can construct $\mathbf{J}_{\mathrm{No}-\mathrm{Ea}, k}^{\mathrm{E}}$ by considering the summation of the terms (14a), (14b), (14c), and (14e), with $U=M$. Moreover, $\mathcal{S}_{R}=\mathcal{S}_{E}=\mathcal{S}_{\text {anchors }}$, so $N_{R}=N_{E}=M$. Substituting the appropriate values of $\alpha, \beta, \gamma$, and $\eta$ from (11)-(13), we easily find that the dominant term in $\mathbf{J}_{\mathrm{No}-\mathrm{Ea}, k}$ scales as $\mathbf{J}_{\mathrm{No}-\mathrm{Ea}, k} \rightarrow M^{2} / 2 \mathbf{I}_{2}$. From the definition of the PEB it follows that $\mathcal{P}_{\mathrm{No}-\mathrm{Ea}} \rightarrow \sqrt{2 / M^{2}}$, and thus $\mathcal{P}_{\mathrm{No}-\mathrm{Ea}} \in$ $\mathcal{O}(1 / M)$

\section{B. Minimum MAC Delay}

Since the eavesdropping measurements require no additional TDMA slots, the MAC delay scales exactly as $\mathcal{M}_{\text {No-Ea }} \in$ $\mathcal{O}(M N)$.

\section{APPENDIX F}

Proof of the No-E CASE

\section{A. $P E B$}

Consider $M$ anchors and $N$ agents uniformly distributed over a fixed two-dimensional area where nodes range only with anchors, but all nodes can eavesdrop. The FIM $\mathbf{J}_{\mathrm{No}-\mathrm{E}}$ consists of $2 \times 2$ block matrices, with $\left[\mathbf{J}_{\mathrm{No}-\mathrm{E}}\right]_{k k}$ given by all terms in (14) and $\left[\mathbf{J}_{\mathrm{No}-\mathrm{E}}\right]_{k l}$ given by (15c), (15d), (15f), and (15h)-(15j) from (15). Now, $\mathcal{S}_{R}=\mathcal{S}_{\text {anchors }}$ and $\mathcal{S}_{E}=$ $\mathcal{S}_{\text {anchors }} \cup \mathcal{S}_{\text {agents }}$, so we use $U=M+N-1$ to determine the constants in (11)-(13). Hence

$$
\left[\mathbf{J}_{\mathrm{No}-\mathrm{E}}\right]_{k l} \rightarrow \begin{cases}\frac{3}{2} M^{2} \mathbf{I}_{2}+\frac{1}{2} M N \mathbf{I}_{2} & k=l \\ -2 M \mathbf{A}\left(\phi_{k l}\right) & k \neq l .\end{cases}
$$

Similarly to Appendix D, exact computation of the EFIM is difficult, so we will construct upper and lower bounds on the FIM $\mathbf{J}_{\text {No-E }}^{\mathrm{L}} \preceq \mathbf{J}_{\text {No-E }} \preceq \mathbf{J}_{\text {No-E }}^{\mathrm{U}}$. Without loss of generality, we focus on agent $k=1$ to analyze the corresponding EFIM lower and upper bounds. 
EFIM Lower Bound: The FIM lower bound $\mathbf{J}_{\text {No-E }}^{\mathrm{L}}$ can be constructed from $\mathbf{J}_{\mathrm{No}-\mathrm{E}}$ by only allowing eavesdropping on ranging transactions involving agent 1 and only letting agent 1 eavesdrop. Inspection of the terms in (14)-(15) leads to a FIM of the form

$$
\left[\mathbf{J}_{\mathrm{No}-\mathrm{E}}^{\mathrm{L}}\right]_{k l} \rightarrow \begin{cases}\frac{3}{2} M^{2} \mathbf{I}_{2}+\frac{1}{2} M N \mathbf{I}_{2} & k=l=1 \\ M^{2} \mathbf{I}_{2} & k=l>1 \\ -2 M \mathbf{A}\left(\phi_{k l}\right) & k=1, l>1 \\ -2 M \mathbf{A}\left(\phi_{k l}\right) & l=1, k>1 \\ \mathbf{0} & k \neq l, k, l>1 .\end{cases}
$$

The EFIM then becomes

$$
\begin{aligned}
& \mathbf{J}_{\mathrm{No}-\mathrm{E}, 1}^{\mathrm{L}, \mathrm{E}} \\
& =\left[\mathbf{J}_{\mathrm{No}-\mathrm{E}}^{\mathrm{L}}\right]_{11}-\sum_{k \in \mathcal{S}_{\text {agents } \backslash\{1\}}}\left[\mathbf{J}_{\mathrm{No}-\mathrm{E}}^{\mathrm{L}}\right]_{1 k}\left[\mathbf{J}_{\mathrm{No}-\mathrm{E}}^{\mathrm{L}}\right]_{k k}^{-1}\left[\mathbf{J}_{\mathrm{No}-\mathrm{E}}^{\mathrm{L}}\right]_{1 k}^{\mathrm{T}} .
\end{aligned}
$$

The second term is given by $4 \sum_{k \in \mathcal{S}_{\text {agents } \backslash\{1\}}} \mathbf{A}\left(\phi_{k 1}\right) \mathbf{A}^{\mathrm{T}}\left(\phi_{k 1}\right) \rightarrow 2 N \mathbf{I}_{2}$. Retaining only the dominant terms, $\mathbf{J}_{\mathrm{No}-\mathrm{E}, 1}^{\mathrm{L}, \mathrm{E}} \rightarrow 3 / 2 M^{2} \mathbf{I}_{2}+1 / 2 M N \mathbf{I}_{2}$.

EFIM Upper Bound: The EFIM upper bound is constructed from $\mathbf{J}_{\mathrm{No}-\mathrm{E}}$ by setting the non-block diagonal elements to zero, i.e., $\left[\mathbf{J}_{\mathrm{N} O-\mathrm{E}}^{\mathrm{U}}\right]_{k l}=0$ for $k \neq l$. The upper bound of the EFIM for agent 1 is given by $\mathbf{J}_{\mathrm{No}-\mathrm{E}, 1}^{\mathrm{U}, \mathrm{E}} \rightarrow \frac{3}{2} M^{2} \mathbf{I}_{2}+\frac{1}{2} M N \mathbf{I}_{2}$.

Final Scaling: Due to the fact that the lower and upper bounds on the EFIM have the same scaling, we conclude that the EFIM itself must have the same scaling. Finally, following the definition of the PEB find that $\mathcal{P}_{\mathrm{No}-\mathrm{E}} \in$ $\mathcal{O}\left(1 / \sqrt{3 M^{2}+M N}\right)$.

\section{B. Minimum MAC Delay}

In our model, anchors can range with agents and other anchors, while the agents eavesdrop. The total number of TDMA slots thus scales as $\mathcal{M}_{\text {No-E }} \in \mathcal{O}\left(M N+M^{2}\right)$.

\section{APPENDIX G}

Proof of the Co-Ea CASE

\section{A. $P E B$}

Consider $M$ anchors and $N$ agents uniformly distributed over a fixed two-dimensional area, where nodes range with all nodes but only anchors can eavesdrop. The FIM $\mathbf{J}_{\mathrm{Co}-\mathrm{Ea}}$ consists of $2 \times 2$ block matrices. Starting from the generalized expressions, the diagonal blocks $(k=l)\left[\mathbf{J}_{\mathrm{Co}-\mathrm{Ea}}\right]_{k k}$ are computed by including terms (14a)-(14c), and (14e) from (14) and the non-diagonal blocks by summing up terms (15a), (15b), (15d), and (15g) from (15). Furthermore, $\mathcal{S}_{R}=$ $\mathcal{S}_{\text {anchors }} \cup \mathcal{S}_{\text {agents }}, \mathcal{S}_{E}=\mathcal{S}_{\text {anchors }}$, and $U=M$ for the constants in (11)-(13). Hence

$$
\left[\mathbf{J}_{\mathrm{Co}-\mathrm{Ea}}\right]_{k l} \rightarrow \begin{cases}\frac{1}{2} M^{2} \mathbf{I}_{2}+\frac{1}{2} M N \mathbf{I}_{2} & k=l \\ 4 \mathbf{A}\left(\phi_{k l}\right) & k \neq l .\end{cases}
$$

In this case it is not possible to remove measurements to determine a lower bound on the FIM. Instead, we note that that (24) behaves similarly to (17): the off-diagonal blocks do not scale with $M$ or $N$, while the diagonal blocks scale at least linear in $M$ and $N$. Hence, for large $M$ and $N$, the diagonal blocks will determine the scaling, and $\mathbf{J}_{\mathrm{Co}-\mathrm{Ea}}^{\mathrm{E}} \rightarrow \frac{1}{2} M^{2} \mathbf{I}_{2}+\frac{1}{2} M N \mathbf{I}_{2}$, so that $\mathcal{P}_{\mathrm{No}-\mathrm{E}} \in \mathcal{O}\left(\sqrt{1 /\left(M^{2}+M N\right)}\right)$.

\section{B. Minimum MAC Delay}

In this case ranging between anchors is not useful, since agents are not allowed to eavesdrop. Hence, only ranging slots between anchors and agents, and between agents are required. This leads to $\mathcal{M}_{\mathrm{Co}-\mathrm{Ea}} \in \mathcal{O}\left(N M+N^{2}\right)$.

\section{APPENDIX H}

\section{Proof of THE Co-E CASE}

Consider $M$ anchors and $N$ agents uniformly distributed over a fixed two-dimensional area where nodes range with all nodes, and all other nodes can eavesdrop. The FIM $\mathbf{J}_{\mathrm{Co}-\mathrm{E}}$ consists of $2 \times 2$ block matrices positioned in row $k$ and column $k$. From the generalized expressions the diagonal blocks $(k=l)\left[\mathbf{J}_{\mathrm{Co}-\mathrm{E}}\right]_{k k}$ are computed by including all terms in (14) and the non-diagonal blocks by including all terms in (15). Furthermore, $\mathcal{S}_{R}=\mathcal{S}_{\text {anchors }} \cup \mathcal{S}_{\text {agents }}$ and $\mathcal{S}_{E}=\mathcal{S}_{\text {anchors }} \cup \mathcal{S}_{\text {agents }}$, and $U=M+N-1$ for the constants in (11)-(13). This leads to

$$
\left[\mathbf{J}_{\mathrm{Co}-\mathrm{E}}\right]_{k l} \rightarrow \begin{cases}2(M+N)^{2} \mathbf{I}_{2} & k=l \\ -2(M+N) \mathbf{A}\left(\phi_{k l}\right) & k \neq l\end{cases}
$$

Comparing (25) with (21), we observe that in both cases the diagonal blocks scale quadratically in $M$ and $N$, while the offdiagonal blocks only scale linearly. Hence, $\mathbf{J}_{\mathrm{Co}-\mathrm{E}}^{\mathrm{E}} \rightarrow 2(M+$ $N)^{2} \mathbf{I}_{2}$, so that $\mathcal{P}_{\mathrm{Co}-\mathrm{E}} \in \mathcal{O}(1 /(M+N))$.

\section{A. Minimum MAC Delay}

In this case, inter-anchor ranging is useful, since agents can eavesdrop. Thus, there will be a ranging transaction between every pair of nodes, leading to $\mathcal{M}_{\mathrm{Co}-\mathrm{E}} \in \mathcal{O}\left((M+N)^{2}\right)$.

\section{REFERENCES}

[1] G. E. Garcia, L. S. Muppirisetty, and H. Wymeersch, "On trade-off between accuracy and delay in cooperative UWB navigation," IEEE Wireless Communications and Networking Conference, pp. 1603-1608, 2013.

[2] _ , "On the trade-off between accuracy and delay in UWB navigation,” IEEE Communications Letters, vol. 17, pp. 39-42, 2012.

[3] K. Pahlavan and X. Li, "Indoor geolocation science and technology," IEEE Communications Magazine, vol. 40, pp. 112-118, 2002.

[4] R. Fontana, E. Richley, and J. Barney, "Commercialization of an ultra wideband precision asset location system," in IEEE Conference on Ultra Wideband Systems and Technologies, 2003, pp. 369-373.

[5] A. Mainwaring, D. Culler, J. Polastre, R. Szewczyk, and J. Anderson, "Wireless sensor networks for habitat monitoring," Proceedings of the 1st ACM international workshop on Wireless sensor networks and applications, pp. 88-97, 2002.

[6] T. Budinger, "Biomonitoring with wireless communications," Annual Review of Biomedical Engineering, vol. 5, pp. 383-412, 2003.

[7] N. Patwari, J. N. Ash, S. Kyperountas, A. O. Hero III, R. L. Moses, and N. S. Correal, "Locating the nodes," IEEE Signal Processing Magazine, vol. 22, pp. 54-59, 2005.

[8] A. F. Molisch, P. Orlik, Z. Sahinoglu, and J. Zhang, "UWB-based sensor networks and the IEEE 802.15.4a standard - a tutorial," International Conference on Communication and Networking in China, 2006.

[9] W. Hirt, "Ultra-wideband radio technology: overview and future research," Computer Communications, vol. 26, pp. 46-52, 2003.

[10] S. Gezici, Z. Tian, G. B. Giannakis, H. Kobayashi, A. F. Molisch, V. H. Poor, and Z. Sahinoglu, "Localization via ultra-wideband radios," IEEE Signal Processing Magazine, pp. 70-84, 2005.

[11] D. Dardari, A. Conti, U. Ferner, A. Giorgetti, and M. Z. Win, "Ranging with ultrawide bandwidth signals in multipath environments," Proceedings of the IEEE, vol. 97, pp. 404-426, 2009.

[12] Y. Shen and M. Z. Win, "Fundamental limits of wideband localization part I: A general framework," IEEE Transactions on Information Theory, vol. 56, pp. 4956-4980, 2010. 
[13] H. Wymeersch, J. Lien, and M. Z. Win, "Cooperative localization in wireless networks," Proceedings of the IEEE, vol. 97, pp. 427-450, 2009.

[14] Y. Shen, H. Wymeersch, and M. Z. Win, "Fundamental limits of wideband localization - part II: Cooperative networks," IEEE Transactions on Information Theory, vol. 56, pp. 4981-5000, 2010.

[15] M. R. Gholami, S. Gezici, and E. G. Ström, "Improved position estimation using hybrid TW-TOA and TDOA in cooperative networks," IEEE Transactions on Signal Processing, vol. 60, pp. 3770-3785, 2012.

[16] Y. Shen, S. Mazuelas, and M. Z. Win, "Network navigation: Theory and interpretation," IEEE Journal on Selected Areas in Communications, vol. 30, pp. 1823-1834, 2012.

[17] M. Z. Win, A. Conti, S. Mazuelas, Y. Shen, W. M. Gifford, D. Dardari, and M. Chiani, "Network localization and navigation via cooperation," IEEE Communications Magazine, vol. 49, pp. 56-62, 2011.

[18] F. Sottile, A. Vesco, R. Scopigno, and M. Spirito, "MAC layer impact on the performance of real-time cooperative positioning," in IEEE Wireless Communications and Networking Conference, 2012, pp. 1858-1863.

[19] C. Lindberg, L. S. Muppirisetty, K.-M. Dahlén, V. Savic, and H. Wymeersch, "MAC delay in belief concensus for distributed tracking," in 10th Workshop on Positioning, Navigation and Communication, 2013.

[20] P. Leone and E. M. Schiller, "Self-stabilizing TDMA algorithms for dynamic wireless ad-hoc networks," International Journal of Distributed Sensor Networks, vol. 2013, p. 17.

[21] T. Wang, Y. Shen, S. Mazuelas, and M. Z. Win, "Distributed scheduling for cooperative localization based on information evolution," IEEE International Conference on Communications, 2012.

[22] D. Satyam, D. Zachariah, A. De Angelis, and P. Handel, "Cooperative decentralized localization using scheduled wireless transmissions," IEEE Communications Letters, vol. 17, pp. 1240-1243, 2013.

[23] M. Rengasamy, E. Dutkiewicz, and M. Hedley, "MAC design and analysis for wireless sensor networks with co-operative localisation," International Symposium on Communications and Information Technologies, 2007.

[24] I. Bucaille, A. Tonnere, L. Ouvry, and B. Denis, "MAC layer design for UWB LDR systems: PULSERS proposal," 4th Workshop on Positioning, Navigation and Communication, 2007.

[25] B. Denis, M. Maman, and L. Ouvry, "On the scheduling of ranging and distributed positioning updates in cooperative IR-UWB networks," International Conference on UWB, pp. 370-375, 2009.

[26] K. Das and H. Wymeersch, "Censored cooperative positioning for dense wireless networks," in IEEE International Symposium on Personal, Indoor and Mobile Radio Communications Workshops (PIMRC Workshops), 2010, pp. 262-266.

[27] — - "Censoring for Bayesian cooperative positioning in dense wireless networks," IEEE Journal on Selected Areas in Communications (JSAC), vol. 30, no. 9, pp. 1835-1842, 2012.

[28] "P400 data sheet," Time Domain Corp., Huntsville, AL, USA.

[29] C. Pedersen, T. Pedersen, and B. H. Fleury, "Exploiting network topology information to mitigate ambiguities in VMP localization," 4th IEEE International Workshop on Computational Advances in MultiSensor Adaptive Processing, pp. 57-60, 2011.

[30] N. Abramson, "The throughput of packet broadcasting channels," IEEE Transactions on Communications, vol. com-25, no.1, pp. 117-128, 1977.

[31] D. Macagnano, G. Destino, F. Esposito, and G. Abreu, "MAC performances for localization and tracking in wireless sensor networks," 4th Workshop on Positioning, Navigation and Communication, 2007.

[32] H. Van Trees, Detection, Estimation and Modulation Theory. Wiley, 1968, vol. 1.

[33] S. M. Kay, Fundamentals of Statistical Signal Processsing: Estimation Theory. Prentice Hall, 1993, vol. 1.

[34] P. Björklund, P. Värbrand, and D. Yuan, "A column generation method for spatial TDMA scheduling in adhoc networks," Ad Hoc Networks, vol. 2, no. 4, pp. 405-418, 2004.

[35] L. Zheng and D. N. Tse, "Diversity and multiplexing: A fundamental tradeoff in multiple-antenna channels," IEEE Transactions on Information Theory, vol. 49, pp. 1073-1096, 2003.

[36] J. T. Isaacs, D. J. Klein, and J. P. Hespanha, "Optimal sensor placement for time difference of arrival localization," in Proceedings of the 48th IEEE Conference on Decision and Control held jointly with the 28th Chinese Control Conference CDC/CCC, 2009, pp. 7878-7884.

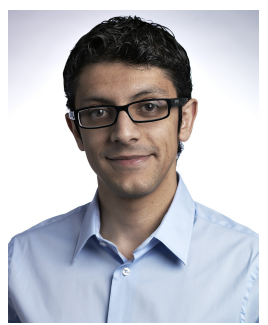

Gabriel E. Garcia received his B.Sc. in Electronics and Communication Engineering from ITESM, Campus Querétaro, Mexico in 2007, his M.Sc. in Communication Engineering, and Licentiate degree in Electrical Engineering, both from Chalmers University of Technology, Sweden, in 2010 and 2013, respectively. Since May 2011, he is pursuing his Ph.D. at the Department of Signals and Systems, Chalmers University of Technology, Gothenburg, Sweden. His research interests include cooperative networks, robust radio frequency localization and navigation, UWB, wireless sensor networks, GNSS, statistical inference and sensor fusion.

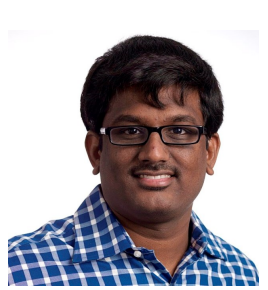

Srikar Muppirisetty received his B.Tech degree in Electronics and Communication Engineering in 2005 from R.V.R \& J.C College of Engineering, India. He received his M.Sc. degree in Communication Engineering in 2009 from Chalmers University of Technology, Sweden. He is currently pursuing a Ph.D. degree at Chalmers University of Technology. $\mathrm{He}$ has over 4 years of industrial experience in physical layer algorithm development for mobile terminals. From 2005-2007 he worked as Member of the Technical Staff at wireless systems division of Digibee Microsystems Pvt. Ltd., Bangalore. During 2009-2012 he worked as a Senior System Software Engineer and as Technical Lead in simulation team at Ericsson (formerly ST-Ericsson) R\&D center, Bangalore. His current research focuses in developing algorithms for resource allocation using position information in wireless communications.

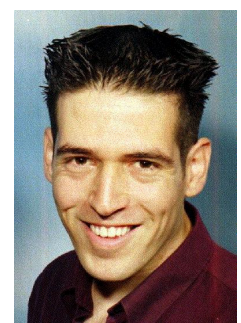

Elad Schiller received his M.Sc., and B.Sc. in Mathematics and Computer Science from Ben-Gurion University of the Negev, Israel and a Ph.D. in Computer Science from the same university. His research excellence has been acknowledged by several highly competitive research fellowships from the Israeli government and the Swedish government. $\mathrm{He}$ is now an associate professor in the Department of Computer Science and Engineering at Chalmers University of Technology. Elad has published in top tier venues (including PODC, DISC, OPODIS, SPAA, SRDS, IEEE-TMC, IEEE-TPDS and Acta Inf.). He has co-authored more than 30 peer revised publications. He served on the program committees for several international conferences, including SSS, PODC, DISC and AlgoSensors. His research interests include distributed computing, with special emphasis on self-stabilizing algorithms, and wireless ad hoc networks.

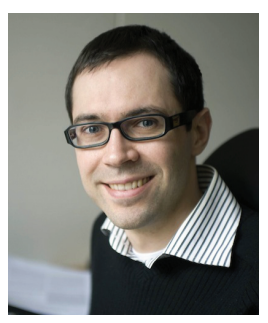

Henk Wymeersch (S'99, M'05) received the Ph.D degree in Electrical Engineering/Applied Sciences in 2005 from Ghent University, Belgium. He is currently an Associate Professor with the Department of Signals and Systems at Chalmers University of Technology, Sweden. Prior to joining Chalmers, he was a Postdoctoral Associate with the Laboratory for Information and Decision Systems at MIT. $\mathrm{He}$ is a member of the IEEE, served as Associate Editor for IEEE COMMUNICATION LETTERS (2009-2013), IEEE TRANSACTIONS ON WIRELESS COMMUNICATIONS (2013-present), and the TRANSACTIONS ON EMERGING TELECOMMUNICATIONS TECHNOLOGIES (2011-present). 